\title{
INERCIA CON FUENTES MATERIALES EN LA DINÁMICA DE PARTÍCULAS
}

\author{
INERTIA WITH MATTER SOURCES \\ IN PARTICLE DYNAMICS
}

\author{
Favio Ernesto, Cala Vitery \\ Físico, Ph.D. Profesor, Departamento de Ciencias Básicas, Universidad Jorge Tadeo Lozano Bogotá, Colombia, \\ Investigador Grupo Matrix. Universidad Militar Nueva Granada, Bogotá, Colombia, \\ E-mail: faviocala@gmail.com. \\ José Amilcar, Rizzo Sierra \\ Físico, Profesor Escuela de física, Universidad Industrial De Santander. Bucaramanga, Colombia. \\ Investigador Grupo Matrix. Universidad Militar Nueva Granada, Bogotá, Colombia, E-mail: amilcar.rizzo@gmail.com. \\ Fecha de recepción: Mayo 05 de 2006. \\ Fecha de aprobación: Enero 25 de 2007.
}

\section{RESUMEN}

Durante los años de gestación de la Teoría General de la Relatividad (TGR) y de la subsiguiente cosmología relativista, Albert Einstein esperaba que su teoría de gravitación satisficiera las ideas de Mach sobre la inercia. Ello ocurría a causa de postular un origen material para la inercia. Se sabe que Einstein encontró dificultades importantes para reconciliar su TGR con el Principio de Mach. En este artículo se discuten los alcances de la teoría alternativa de gravitación clásica de J. Barbour y B. Bertotti. Ésta pretende incorporar en forma consistente el Principio de Mach en el contexto de la dinámica clásica. Su relevancia conceptual y heurística es discutida.

PALABRAS CLAVE: Inercia, Principio de Mach, Gravitación, Cosmología.

\begin{abstract}
While creating his General Theory of Relativity $(G R)$ and the subsequent relativistic cosmology, Einstein hoped that this new theory of gravitation would conform to Mach's ideas on inertia. In doing so, he faced important difficulties trying to ensure $G R$ 's accordance with the material origin of inertia. The scope of Barbour and Bertotti's alternative gravitation theory is examined. This theory intends to incorporate Mach's principle consistently in the context of classical dynamics. It's conceptual and heuristic relevance is discussed.
\end{abstract}

KEY WORDS: Inertia, Mach's Principle, Gravitation, Cosmology. 


\section{INTRODUCCIÓN}

En 1883, Ernst Mach propuso que la inercia de un cuerpo debía tener fuentes materiales. La materia estelar, en lugar del invisible espacio absoluto, debía originar el conjunto de fuerzas inerciales típicas de la mecánica clásica. La idea durante largo tiempo se convirtió en un fuerte estímulo para Einstein. Para Mach una formulación inteligible de la inercia pasaba por derivar las familias de sistemas inerciales a partir de la materia estelar. Sin embargo, Einstein, en lugar de derivar los sistemas inerciales, intentó eliminarlos y encontró importantes dificultades para llevar a buen concurso las ideas de Mach sobre la inercia. En este artículo se discuten los alcances de la teoría de Barbour y Bertotti (1977), teoría que pretende guardar mayor coherencia y fidelidad con el relacionismo de Mach ${ }^{1}$.

\section{DINÁMICA DE PARTÍCULAS}

Antes de Einstein, si a algo se le podía llamar el principio de Mach era a la consigna de que las familias de sistemas inerciales empíricamente aceptables debían derivarse del conjunto de interacciones relativas entre los puntos materiales que componen el universo entero. Esto debía conducir a una dinámica de partículas inteligible en el espíritu relacional. Se sabe que Einstein no tomó este camino. Dejamos a Einstein y seguimos ahora a Barbour en su intento por dar continuidad al principio de Mach en el contexto clásico de partículas.

La idea original de Barbour (1974) y Barbour y Bertotti (1977) fue considerar al principio de Mach como el requerimiento de que las leyes de la dinámica fuesen invariantes bajo la acción del grupo:

$$
\begin{aligned}
& x^{i} \rightarrow \bar{x}^{i}=R_{j}^{i} x^{j}(\lambda)+a^{i}(\lambda), \quad i, j=1,2,3 \\
& \lambda \rightarrow \bar{\lambda}=F(\lambda), \quad\left(\frac{d F}{d \lambda}>0\right)
\end{aligned}
$$

donde $\lambda$ es un parámetro temporal arbitrario utilizado para tabular configuraciones instantáneas y $R_{j}^{i} x^{j}(\lambda)$ es una matriz ortogonal dependiente del parámetro temporal $y$ las $a^{\alpha}(\lambda)$ son funciones suaves dependientes de $\lambda$. Barbour llama a este el grupo de Leibniz, pero es preferible — siguiendo a Earman (1989 p.27) - llamarlo el grupo de Mach. Básicamente porque la condición (2) es flexible respecto a la definición del tiempo y no presupone una métrica temporal exterior absoluta. El tiempo puede surgir como una abstracción derivada de la propia dinámica ${ }^{2}$

1) Por relacionismose entiende la consigna de que el espacio - y el tiempo - es una abstracción derivada del conjunto de relaciones entre objetos materiales y no una entidad física real (substancial) independiente de éstos como pensaba Newton.

2) Rara vez se menciona a Mach en este sentido. Por ello acaso sea conveniente recordar sus palabras al respecto (Mach 1883, p.272):

"It is utterly beyond our power to measure the change of things by time. Quite the contrary, time is an abstraction, at which we arrive by means of the changes of things; made because we are not restricted to any definite measure, all- 
La invariancia ante la condición (1) es la expresión matemática para la aplicación del principio de Identidad de los Indiscernibles (PII) de Leibniz a un conjunto de configuraciones relativas equivalentes flotando (o gravitando) en un espacio métrico Euclideano $\left(E^{3}\right)$. Nótese que para que ésta aplicación de PII sea efectiva, las transformaciones $x^{\alpha} \rightarrow \bar{x}^{\alpha}$ deben ser interpretadas como transformaciones pasivas, como una mera renominación de puntos. La idea es que representaciones matemáticas de configuraciones instantáneas relativas equivalentes pero rotadas globalmente ( $\pi$, por ejemplo) o desplazadas (3 metros hacia el este -por ejemplo-) correspondan a la misma configuración física.

Barbour y Col. estudian teorías invariantes bajo el grupo de Mach que puedan derivarse de un principio variacional. Para ello consideran teorías cuya acción,

$$
J=\int d \lambda L,
$$

resulte invariante ante las simetrías (1) y (2) y en las que además $L$ sea un Lagrangiano de la forma $L=V T$ (no $L=V+T$, como el Lagrangiano estándar).

En la más prometedora de estas teorías (Barbour y Bertotti 1977) escogen:

$$
\begin{aligned}
& V=\sum_{i<j}^{N} \frac{m m_{j}}{r_{i j}} \\
& T=\left(\sum_{i<j}^{N} m_{i} m_{j} \dot{r}_{i j}^{2}\right)^{\frac{1}{2}}
\end{aligned}
$$

Donde

$$
\dot{r}_{i j}=\frac{d r_{i j}}{d \lambda}
$$

Lo que debe hacer especialmente Machiana esta teoría en comparación con una Leibniziana, es - tal como se mencionaba anteriormente- la posibilidad de definir una métrica temporal desde la propia dinámica. Es más probable que para un relacionista Leibniziano la medida del cambio relativo entre configuraciones espaciales sucesivas pueda estar tabulada por un tiempo absoluto (i.e., $d \lambda=d t$ ). Sin embargo, los detalles históricos sobre esta interpretación pueden ser discutibles. No así en el caso de Mach, quien explícitamente considera al tiempo como una medida del

being interconnected. A motion is termed uniform in which equal increments of space described correspond to equal increments of space described by some motion with which we form a comparison, as the rotation of the earth. A motion may, with respect to another motion be uniform. But the question whether a motion is in itself uniform, is senseless. With just a little justice, also, may we speak of an 'absolute time' —of a time independent of change-. This absolute time can be measured by comparison with no motion; it is neither a practical nor scientific value; and no one is justified in saying he knows aught about it. It is an idle metaphysical conception.»

[Énfasis en cursiva del original]. 
cambio facilitada por la regularidad relativa entre ciertos movimientos (como el de la rotación de la tierra con respecto a las estrellas distantes).

En esta línea argumental, Barbour y Bertotti emplean una definición cosmológica para el tiempo, así:

$$
d \lambda=d t=\left(\sum_{i<j}^{N} m_{i} m_{j} \dot{r}_{i j}^{2}\right)^{2}
$$

De modo que

$$
T \equiv 1
$$

La expresión (6), que usualmente se tomaría por una imposición sobre la energía cinética del sistema, es interpretada como la definición dinámica de la métrica temporal. Esta es una expresión explícita de la aseveración de que el tiempo es una medida del cambio. Puede mostrarse que en términos de la masa del universo $M$ y su radio $R, T \propto M R^{2}$. De aquí Barbour y Bertotti llegan a la elegante conclusión de que el universo es su propio reloj (Ibíd. p. 5).

Para esta teoría (BB1) las ecuaciones de movimiento (las ecuaciones de EulerLagrange) expresadas con respecto al tiempo cósmico $\tau$ son:

$$
V \sum_{j \neq i} \frac{m_{j}}{r_{i j}} \ddot{r}_{i j}=-\sum_{j \neq i} \frac{m_{j}}{r_{i j}^{2}}+\frac{V}{2} \sum_{j \neq i} \frac{m_{j}}{r_{i j}^{2}} \dot{r}_{i j}^{2}-V \sum_{j \neq i} \frac{m_{j}}{r_{i j}} \dot{r}_{i j}
$$

En el espíritu relacional, el Lagrangiano y las ecuaciones de movimiento dependen de distancias puramente relativas y sus derivadas $L=L\left(r_{i j}, \dot{r}_{i j}\right)$ Por esto la teoría es mejor formulada en un espacio de configuración relativa $T \times \tilde{Q}$

En el caso de una partícula 'libre', el espacio de configuración relativa no existe y no hay teoría de movimiento. En el caso de que existan tan sólo dos partículas en el universo, lo único que puede determinarse es si éstas se alejan o se aproximan. 'Pero ningún significado puede asignarse a la velocidad con que lo hacen pues el tiempo es este movimiento relativo'. (Ibíd. p. 6). Cuando se estudian tres (o más) cuerpos, Barbour y Bertotti encuentran que ciertas configuraciones -en particular aquellas en que $N$ cuerpos son coplanares - no permiten una descripción unívoca de su movimiento relativo futuro. El problema de valor inicial no puede formularse satisfactoriamente en todos los casos. De hecho, en el caso de los tres cuerpos se ven obligados e introducir 'fronteras' para redefinir el problema de valor inicial más allá de las regiones en que las soluciones no son físicamente aceptables.

A estas alturas alguien podría preguntarse qué sentido tiene una teoría que soluciona satisfactoriamente el problema relacional para uno o dos cuerpos, pero que para un número finito de partículas autogravitantes encuentra serias limitaciones en comparación con la teoría de Newton. Sin embargo, el relacionista puede alegar que nunca han sido observados 3 cuerpos completamente aislados en un universo vacío o que un universo cuyas partículas se distribuyen coplanarmente es altamente 
improbable, de hecho, ni siquiera empíricamente correcto. Al fin y al cabo, el principio de Mach cobra su mejor sentido en un contexto cosmológico. Así que resulta conveniente intentar definir una dinámica local causalmente relacionada con un entorno cosmológico adecuado y sólo modelos de universo tipo isla finitos pueden ser contemplados consistentemente.

Barbour y Bertotti estudian la dinámica de un sistema local de $n$ partículas ubicadas cerca del centro de un cascarón esférico delgado de masa $M$ y radio $R$ en reposo. La simetría esférica está motivada por razones de consistencia observacional y simplicidad. El origen de coordenadas está puesto en su centro. En el límite cosmológico,

$$
\frac{r_{i}}{R} \rightarrow 0, \frac{m_{i}}{M} \rightarrow 0, \frac{r_{i} M}{R m_{i}} \rightarrow \text { finito }
$$

donde $r_{i}$ y $m_{i}$ son respectivamente las coordenadas y las masas de las partículas que componen el sistema local. El Lagrangiano local toma la forma:

$$
L_{l o c}=\frac{1}{2} \sum_{i}|\dot{r}|^{2}+\frac{4 R \dot{R}^{2}}{M} \sum_{i<j} \frac{m_{i} m_{j}}{r_{i j}}+\frac{3 R}{2 M} \sum_{i<j} \frac{m_{i} m_{j}}{r_{i j}} \dot{r}_{i j}^{2}
$$

Donde el punto denota la diferenciación con respecto al tiempo local t, relacionado con el tiempo cósmico $\tau$ por $d t \propto R^{2} d t$

El primer resultado interesante es que dentro del limite cosmológico (7), el Lagrangiano (8) es invariante ante transformaciones de Galileo. Esto es importante ya que la invariancia Galileana local es derivada de una dinámica global invariante ante el grupo de Mach. La dinámica cosmológica relacional produce la apariencia local de una dinámica al estilo de la newtoniana. Esto permite hablar localmente de fuerzas inerciales, de fuerzas centrífugas y de partículas libres, aunque queda claro que este tipo de movimientos están mediados por la influencia causal de la materia estelar distante. La respuesta de Barbour y Bertotti al desafío epistemológico planteado por Mach parece contundente - al menos para un universo altamente simétrico como el propuesto-(Ibíd. p. 12):

"If the local dynamics is subjected to a transformation (1) corresponding to an accelerated and rotated frame of reference, the usual apparent forces show up in the equations of motion; since, however, (8) is derived from a relative configuration dynamics, just the same forces are obtained if one starts from an accelerated and rotating mass shell. The Machian origin of apparent forces is now clear and explicit."

Aquí resulta casi inevitable imaginar el universo rotando alrededor del balde de Newton $^{3}$. Dentro del límite en que $\chi=R m / M r<1$, el tercer término de (8) puede

3) Se refiere al bien conocido experimento mental del Escolio de los Principia de Newton. Éste fue ideado, en parte, para imaginar el espacio absoluto (sustancialista) de Newton. 
ser despreciado y las ecuaciones de movimiento locales son idénticas a las de Newton, pero con una constante cosmológica $G=4 R \dot{R}^{2}$. Esto permite a Barbour y Bertotti hacer un ajuste apropiado de los valores de $R, \dot{R}$ y $M$ consistente con los valores conocidos para el avance del perihelio de mercurio. Las leyes de gravitación e inercia de Newton son recuperadas localmente con una pequeña corrección que permite mejorar su dinámica planetaria. La restricción $\chi \ll<1$ no es una camisa de fuerza de la teoría. Al levantarla, se ve que el tercer término de (8) es el responsable local de los efectos Machianos de la teoría. Al igual que Einstein (1912, 1918), Barbour y Bertotti consideran la influencia de un cascarón esférico de radio $R_{0}$ y masa $M_{0}$ sobre una partícula de prueba ubicada cerca de su centro (que en este caso, a su vez coincide con el centro del cascarón cosmológico, ¡con el centro del universo!). Y al igual que Einstein encuentran que: (a) la presencia de materia cercana $\left(M_{0}\right)$ aumenta la masa inercial de un cuerpo y (b) que la rotación del cascarón $R_{0}$ induce un arrastre inercial recuperando el efecto de arrastre parcial de Lense-Thirring (1918) para TGR.

La ventaja comparativa de los cálculos de Barbour y Bertotti radica en que con ellos se prescinde de la estructura inercial absoluta del espacio exterior al cascarón en rotación. Esto conllevaba, en el caso de Lense-Thirring, a la agridulce conclusión de que la materia arrastraba parcialmente los marcos inerciales dejando parte de la estructura inercial anclada al espacio exterior (Cáp. 3). En este caso el arrastre parcial es justificado por la presencia exterior de materia distante en el cascarón cosmológico, un resultado perfectamente Machiano.

Parece claro que Brans (1962) mostró que (a) ${ }^{4}$ no es más que un efecto de coordenadas en TGR, y por otra parte, la efectividad predictiva de (b) espera ser comprobada empíricamente con los giroscopios de Gravity Probe- $\mathrm{B}^{5}$. Para (a) podrían alegarse las mismas dificultades que en TGR para detectar un aumento local de la masa inercial, a saber, la insignificancia relativa de la presencia de nueva materia una galaxia, por ejemplo- en la vecindad de un cuerpo comparada con la materia total del universo. Sin embargo, prescindiendo de la altísima simetría que obliga a ubicar las partículas de prueba o el sistema solar cerca del centro del universo, la teoría también predice indeseables efectos anisotrópicos sobre la masa inercial de un cuerpo que no pueden ser justificados de igual manera ${ }^{6}$. Esta es la gran desventaja experimental de la teoría al compararla con la teoría substancialista de Newton.

4) Véase Cala, F. E. (2005) para un estudio detallado al respecto.

5) En este sentido véanse en http://einstein.stanford.edu, detalles sobre Gravity Probe-B, un experimento desarrollado por la NASA y la universidad de Stanford destinado a verificar, de acuerdo con TGR, el arrastre inducido por la rotación de la tierra sobre 4 giroscopios puestos a orbitar en un satélite a 400 millas de altura (directamente sobre los polos terrestres).

6) El experimento de Hughes-Drever, con espectroscopía nuclear, pone un límite superior de $10^{-23}$ a este tipo de anisotropías. BB1 predice anisotropías de masa potencialmente detectables del orden de $10^{-8}$. 
Aquí la clave pasa por saber cuán capaz de rivalizar con la de Newton resulta esta teoría. Aparte de los indeseables efectos anisotrópicos, que deberían servir de estimulo para intentar algún tipo de corrección apropiada para la forma del Lagrangiano, la teoría tiene otra desventaja significativa inherente al propio enfoque relacional y esto sí que podría resultar hiriente. Se trata del problema de valor inicial. Earman lo pasa por alto, pero la atención prestada a éste por Barbour y Col. les lleva a desarrollar un nuevo principio relacional que puede ser extendido a teorías de campo relativistas.

Volviendo al problema de valor inicial, debido a la invariancia ante el grupo de Mach ((1)+(2)) las ecuaciones de movimiento contienen siete funciones arbitrarias de $\lambda$. La arbitrariedad de estas funciones, que en general contienen información sobre la energía, el momento lineal y el momento angular de un sistema aislado de $N$ partículas, es el resultado directo de las simetrías de las ecuaciones de movimiento (Teorema de Noether). Como consecuencia de ello, no existe una forma objetiva de definirlas. Todo esto seria irrelevante si parte de esta información no resultara determinante en la evolución futura del sistema. Nos gustaría un comportamiento similar al de la dinámica newtoniana puesto que allí podemos escoger el origen de coordenadas y su velocidad -las constantes arbitrarias de integración-sin afectar la descripción física futura de un sistema newtoniano. En la dinámica relacional parte de la información arbitraria resulta determinante, así que hay que añadir importantes restricciones adicionales para determinar univocamente la evolución del sistema.

Esto último puede resultar chocante para algunos relacionistas. El argumento es que si se sigue consistentemente a Leibniz, para determinar unívocamente la evolución de un sistema aislado debería bastar con la información sobre la configuración relativa instantánea y su velocidad relativa de cambio (si además se sigue a Mach, la métrica temporal, indispensable para tabular la velocidad relativa, debería estar determinada por el propio sistema) ${ }^{7}$.

El relacionismo per se no debería implicar determinismo. Pero el determinismo de la física de Newton enfrenta al relacionista a un grueso dilema. O se limita a proporcionar la misma información inicial requerida por la dinámica newtoniana y construye una teoría que admite la evolución no determinista de sistemas de partículas autogravitantes, o, para salvar el determinismo, impone restricciones adicionales

7) Barbour y Bertotti (1977) resuelven el problema de Cauchy asi:

"Given a relative configuration and the direction in which a configuration is moving in the relative configuration space, choose an arbitrary particle as the origin of the Cartesian coordinate system for all times. Take two other particles and and require the $x y$ - plane always coincide with the plane defined by , and and that $j$ always lie on the - axis. This fixes six of the arbitrary functions. The seventh is fixed by choosing a definite time parameter. The motion is then uniquely defined by the initial conditions. $"$

La cursiva es nuestra y corresponde a toda la información adicional requerida para resolver el problema del valor inicial. 
que restringen considerablemente el tipo de sistemas que pueden ser consistentemente estudiados. Desde luego parece más sano escoger lo segundo, pero en cualquier caso esta alternativa supone limitaciones en el poder predictivo de la dinámica relacional. Los sistemas mal comportados podrían ser considerados físicamente inaceptables por la teoría, pero hace falta algún tipo de justificación teórica o empírica más fuerte para descartarlos de golpe.

\section{CONCLUSIÓN}

Resumiendo, Barbour y Bertotti han construido una teoría clásica que desde el punto de vista formal es completamente relacional y Machiana y que mejora la dinámica planetaria de Newton, pero que a su vez predice efectos anisotrópicos físicamente inaceptables. La teoría también presenta limitaciones en relación con el problema de valor inicial. En todo caso se insiste en este artículo en la lección formal que Barbour y Bertotti pretenden haber sentado. Esta es: tras 300 años de desarrollo convencional de física clásica espacio-temporal substancialista, por primera vez una teoría relacional parece haberse levantado del suelo. Leibniz, Berkeley e incluso Mach habían defendido su relacionismo a nivel epistemológico sin llegar a proporcionar una teoría cuantitativa capaz de rivalizar con la de Newton. La importancia heurística de esta teoría radica en que el formalismo pretende sentar las bases para extender su aplicación a la arena de las teorías de campo unificado. Barbour ha sido parcialmente exitoso al llevar esta tarea al contexto de la Geometrodinámica Relativista (Barbour 1994, 2003).

\section{REFERENCIAS BIBLIOGRAFICAS}

[1] BARBOUR, J.B. (1974) 'Relative-Distance Machian Theories' Nature, 249, pp. 328-329. Erratum 250, p. 606.

[2] BARBOUR, J. B., y Bertotti, B. (1977) 'Gravity and Inertia in a Machian Framework' II Nuovo Cimento B, 38, pp. 1-27.

[3] BARBOUR, J.(1994) 'The timelessness of quantum gravity: I The evidence from the classical theory, II The appearance of dynamics in static configurations'. Class. and Quant. Grav 11, pp. 2853.

[4] BARBOUR, J. B. (2003) 'Dynamics of Pure Shape, Relativity and the Problem of Time', E-print disponible en arXiv:gr-qc/0309089.

[5] BRANS, C. (1962) 'Mach's Principle and the Locally Measured Gravitational Constant in General Relativity', Physical Review, 125, pp. 388-396.

[6] CALA, F. E. (2005) 'ElAumento de la Masa Inercial: Einstein y las Coordenadas', Ciencia e Ingeniería Neogranadina, 15, pp 116-127. 
[7] EARMAN, J. (1989) World Enough and Space-Time. Cambridge, Massachusetts. MIT Press.

[8] EINSTEIN, A. (1912) 'Gibt es eine Gravitationswirkung die der elektrodynamischen Induktionswirkung analog ist?', Vierteljahrschrift f"ur Gerichtline Medizin, 44, 37-40.

[9] EINSTEIN, A. (1918) 'Prinzipielles zur allgemeinen Relativitätstheorie', Annalen derPhysik, 55, pp. 241-244.

[10] LENSE, J. and Thirring, H. (1918) 'Über den Einfluss der Eigenrotation der Zentralkörper auf die Bewegung der Planeten und Monde nach der Einsteinschen Gravitationstheorie', Physikalische Zeitschrift, 19, pp. 156-163 [On the Influence of the Proper Rotation of Central Bodies on the Motions of Planets and Moons According to Einstein's Theory of Gravitation].

[11] MACH, E. (1883) The Science of Mechanics. La Salle, Illinois. 1960. Trad. de T.J. Mc. Cormack. Open Court. 